\title{
Prolonged muscle vibration increases stretch reflex amplitude, motor unit discharge rate, and force fluctuations in a hand muscle
}

\author{
Minoru Shinohara, Chet T. Moritz, Michael A. Pascoe, and Roger M. Enoka \\ Department of Integrative Physiology, University of Colorado at Boulder, Boulder, Colorado
}

Submitted 16 March 2005; accepted in final form 14 July 2005

\begin{abstract}
Shinohara, Minoru, Chet T. Moritz, Michael A. Pascoe, and
Roger M. Enoka. Prolonged muscle vibration increases stretch reflex amplitude, motor unit discharge rate, and force fluctuations in a hand muscle. J Appl Physiol 99: 1835-1842, 2005. First published July 14, 2005; doi:10.1152/japplphysiol.00312.2005.-The purpose of this study was to compare the influence of prolonged vibration of a hand muscle on the amplitude of the stretch reflex, motor unit discharge rate, and force fluctuations during steady, submaximal contractions. Thirty-two young adults performed 10 isometric contractions at a constant force $(5.0 \pm 2.3 \%$ of maximal force) with the first dorsal interosseus muscle. Each contraction was held steady for $10 \mathrm{~s}$, and then stretch reflexes were evoked. Subsequently, 20 subjects had vibration applied to the relaxed muscle for $30 \mathrm{~min}$, and 12 subjects received no vibration. The muscle vibration induced a tonic vibration reflex. The intervention (vibration or no vibration) was followed by 2 sets of 10 constant-force contractions with applied stretches (After and Recovery trials). The mean electromyogram amplitude of the short-latency component of the stretch reflex increased by $33 \%$ during the After trials $(P<0.01)$ and by $38 \%$ during the Recovery trials $(P<$ $0.01)$. The standard deviation of force during the steady contractions increased by $21 \%$ during the After trials $(P<0.05)$ and by $28 \%$ during the Recovery trials $(P<0.01)$. The discharge rate of motor units increased from $10.3 \pm 2.7$ pulses/s (pps) before vibration to $12.2 \pm 3.1 \mathrm{pps}(P<0.01)$ during the After trials and to $11.9 \pm 2.6$ pps during the Recovery trials $(P<0.01)$. There was no change in force fluctuations or stretch reflex magnitude for the subjects in the Control group. The results indicate that prolonged vibration increased the short-latency component of the stretch reflex, the discharge rate of motor units, and the fluctuations in force during contractions by a hand muscle. These adjustments were necessary to achieve the target force due to the vibration-induced decrease in the force capacity of the muscle.
\end{abstract}

first dorsal interosseus muscle; Ia afferents; steadiness

VIBRATION HAS BEEN APPLIED to the skeletal muscle and tendon to manipulate the excitatory synaptic input from group Ia afferents onto $\alpha$-motor neurons in both physiological studies and clinical practice. With an acute application of vibration to a relaxed muscle, muscle spindles in humans exhibit a one-toone response to each cycle of vibration up to $\sim 100 \mathrm{~Hz}(3,30)$, which can evoke a tonic vibration reflex $(7,21)$. Sustained vibration, however, depresses the amplitude of the $\mathrm{H}$ reflex, probably due to a decline in the release of transmitter from the terminals of group Ia afferents $(1,12)$. Prolonged vibration also appears to reduce the fluctuations in force that occur during steady, submaximal contractions $(6,38,40)$.

Force fluctuations during steady contractions are profoundly influenced by the discharge rate variability of motor units (17, 26). It is unknown, however, how the alteration in the Ia

Address for reprint requests and other correspondence: M. Shinohara, Clare Rm. 114, 354 UCB, Dept. of Integrative Physiology, Univ. of Colorado, Boulder, CO 80309-0354 (e-mail: shinohara@colorado.edu). afferent input modulates motor unit activity during submaximal contractions. Hence, the present study examined the effect of perturbing Ia afferent feedback with prolonged muscle vibration on motor unit activity and force fluctuations. It is possible that prolonged vibration depresses the Ia afferent input onto $\alpha$-motor neurons and that this change in synaptic input decreases the discharge rate variability of motor units.

The purpose of this study was to compare the influence of prolonged vibration of a hand muscle on the amplitude of the stretch reflex, motor unit discharge rate, and force fluctuations during steady, submaximal contractions. Muscle vibration was employed instead of tendon vibration to avoid the potential confounding influences of the Ib afferents and because tendon vibration of the first dorsal interosseus muscle was not effective at evoking a tonic vibration reflex at amplitudes that did not cause discomfort. It was hypothesized that prolonged vibration would decrease the amplitude of the stretch reflex, reduce discharge variability of motor units, and diminish the force fluctuations during steady contractions. Some of the data have been presented in abstract form (33).

\section{METHODS}

Experiments were performed on the left hand of 32 adults (15 men, 17 women; mean \pm SD: $21.9 \pm 3.5 \mathrm{yr}$; range: $18-30 \mathrm{yr}$ ). All subjects reported an absence of neuromuscular disorders and were right-hand dominant, as quantified by the laterality quotient of the Edinburgh Handedness Inventory (28), and they did not participate in activities that involved skilled movements with either hand. Twenty subjects received vibration of the first dorsal interosseus muscle (Vibration group), and 12 subjects performed the same tasks as those in the Vibration group but did not receive vibration (Control group). The laterality quotient of the subjects was similar for the Vibration $(0.832 \pm 0.138)$ and Control $(0.833 \pm 0.207)$ groups $(P=0.98)$. The Institutional Review Board at the University of Colorado approved the experimental procedures, and all subjects gave informed consent before participation in the study.

\section{Experimental Arrangement}

The experiments were performed on the first dorsal interosseus muscle of the left hand. The experimental arrangement for measuring the muscle activity and force was identical to a previous study on force fluctuations with this muscle (32). The subject was seated in a shielded experimental room and faced a video display monitor that was located $1.2 \mathrm{~m}$ in front of the subject. The arm was abducted $\sim 0.5$ $\operatorname{rad}\left(\sim 30^{\circ}\right)$ and the elbow flexed to a right angle with the hand and forearm pronated and resting on separate supports. The index finger was fully extended in the horizontal plane, and the other three fingers were flexed around a semicircular grip (Fig. 1). The thumb was extended in a horizontal position and held with a brace. The index

The costs of publication of this article were defrayed in part by the payment of page charges. The article must therefore be hereby marked "advertisement" in accordance with 18 U.S.C. Section 1734 solely to indicate this fact. 


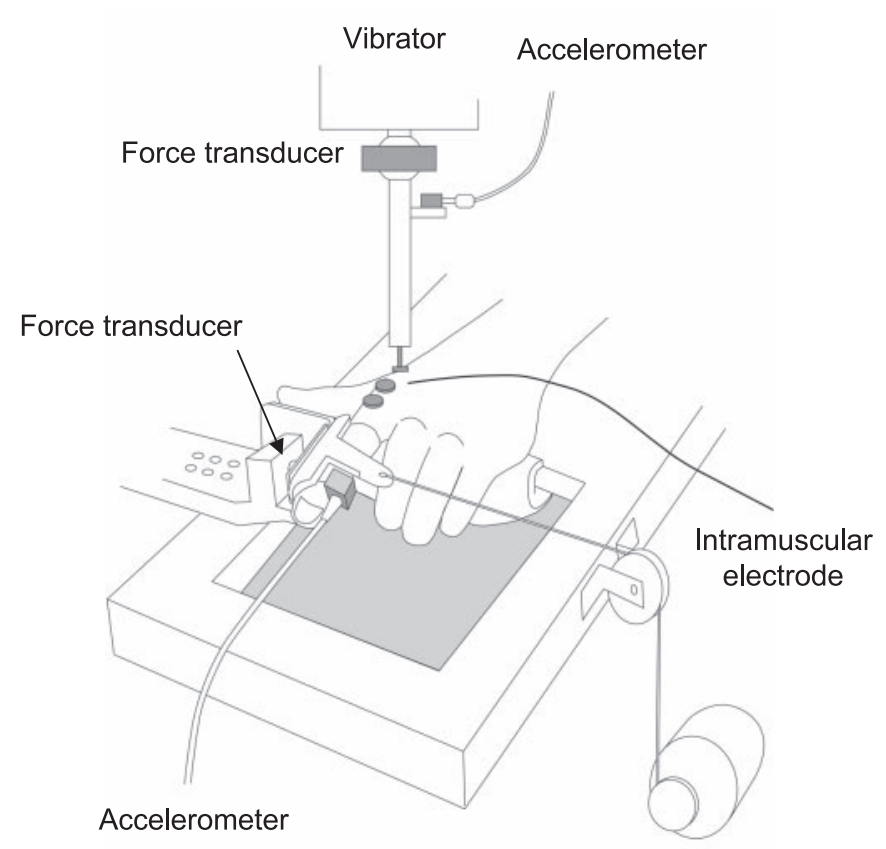

Torque motor

Fig. 1. Schematic drawing of the experimental arrangement. Subjects performed isometric contractions with the first dorsal interosseus muscle, and the abduction force exerted by the extended index finger was measured with a force transducer. A torque motor was used to evoke stretch reflexes as subjects performed steady, submaximal contractions. An accelerometer on the finger identified the onset of the stretch stimulus. A force transducer and an accelerometer on the shaft of the vibrator measured the force and amplitude of the vibration applied to the first dorsal interosseus muscle. Motor unit potentials were recorded with an intramuscular electrode.

finger was placed in a semicircular splint that was positioned on the lateral surface of the index finger. With this arrangement, abduction of the index finger at the metacarpophalangeal joint was produced almost exclusively by contraction of the first dorsal interosseus muscle (22).

With the index finger abducted $\sim 0.1 \mathrm{rad}\left(5^{\circ}\right)$ from the neutral position, subjects performed isometric contractions with the first dorsal interosseus muscle. A force transducer (model 13, Sensotec, Columbus, $\mathrm{OH} ; 0.045 \mathrm{~V} / \mathrm{N}$ and $0.45 \mathrm{~V} / \mathrm{N}$ ) detected the abduction force exerted at the proximal interphalangeal joint of the index finger. An accelerometer (model 7265A, Endevco, San Juan Capistrano, CA) was attached to the proximal interphalangeal joint of the index finger to record acceleration in the abduction-adduction plane during muscle stretches.

A bipolar, fine-wire electrode was inserted into the first dorsal interosseus muscle to record the motor unit potentials. The electrode consisted of three Formvar-insulated, stainless steel wires $(50-\mu \mathrm{m}$ diameter; California Fine Wire, Grover Beach, CA) that were held together at the recording tip. The wires were threaded through the lumen of a disposable 27-gauge hypodermic needle, and a barb of $\sim 2$ $\mathrm{mm}$ in length was created at the tip of the recording end of the electrode. The electrode was implanted into the muscle to a depth of $10-20 \mathrm{~mm}$, and the needle was withdrawn, leaving the wires within the belly of the muscle. Recordings were obtained from two wires, amplified $(\times 5,000)$, and band-pass filtered $(100-8,500 \mathrm{~Hz}$; S-series, Coulbourn Instruments, Allentown, PA). Motor units were detected online using a time-amplitude window discriminator (Dual comparator/window discriminator V21-10, Coulbourn Instruments) and displayed on an oscilloscope.

The surface electromyogram (EMG) of the first dorsal interosseus muscle was recorded with bipolar electrodes ( $\mathrm{Ag}-\mathrm{AgCl}, 4-\mathrm{mm}$ diameter; $\sim 25 \mathrm{~mm}$ center to center) that were secured to the skin overlying the muscle. One electrode was placed over the belly of the muscle, and the other electrode was attached to the skin over the base of the proximal phalanx of the index finger $(31,32)$. A common electrode (Ag-AgCl, 4-mm diameter) was placed on the styloid process of the ulna on the dorsal surface of the hand. The surface EMG signals were amplified $(\times 1,000)$ and band-pass filtered $(13-1,000 \mathrm{~Hz}$; V75-04, Coulbourn Instruments).

A torque motor (PMA44Q, Pacific Scientific, Rockford, IL) was used to stretch the first dorsal interosseus muscle by inducing a rapid adduction of the index finger (Fig. 1). The torque motor was attached to a string that was directed over a pulley and connected to the finger splint at the proximal interphalangeal joint. Custom-designed software written in MatLab (version 6.5, The Mathworks, Natick, MA) was used to control the torque motor.

An electromagnetic vibrator (model V203, Ling Dynamic Systems, Yalesville, CT) was used to apply vibration to the muscle belly. A force transducer (model MLP-10, Transducer Techniques, Temecula, CA) was placed in series with a nonconductive Micarta rod (Fig. 1). The baseline force was adjusted by changing the length of an aluminum cylinder that made contact with the skin at the end of the rod. An accelerometer (model 7265A, Endevco, San Juan Capistrano, CA) was mounted on the rod to monitor the vibration.

Motor unit recordings were digitized at 20,000 samples/s, and the index finger abduction force, acceleration, and the surface EMG were digitized at 1,024 samples/s (Power 1401, Cambridge Electronic Design, Cambridge, UK). These data were stored on a personal computer for later analyses.

\section{Experimental Procedures}

The subjects were asked to perform three tasks with the left index finger: 1) a maximal voluntary contraction (MVC) in the abduction direction, 2) a gradual increase in muscle force to identify the recruitment threshold of a motor unit, and 3) steady isometric contractions at a constant force. Vibration was applied to the relaxed first dorsal interosseus muscle of subjects in the Vibration group after 10 trials of the steady contractions.

MVC task. The MVC involved a gradual increase in the abduction force exerted by the index finger from baseline to maximum in 3-4 s and then sustained at maximum for 1-2 s. The index finger force was displayed on the monitor. The timing of the task was based on a verbal count given at 1-s intervals, with vigorous encouragement from the investigators when the force began to plateau. Each subject performed three MVC trials, with subsequent trials performed if the difference in peak force between two MVCs was $>5 \%$. The trial with the highest peak force was chosen for analysis. Rest periods of $>60 \mathrm{~s}$ were given between each MVC trial. This task was performed at the start and end of the experiment.

Recruitment threshold. The recruitment threshold of the motor unit was determined by a ramp contraction task $(26,35)$. The task began by the subject gradually increasing the contraction intensity as the investigators observed the intramuscular EMG signal for the appearance of a candidate motor unit. The subject was provided with visual feedback of abduction force and audio feedback of the discharge for the motor unit being tracked. Once a unit had been identified, the subject was instructed to increase the force gradually until the motor unit became active and then to reduce the force slowly to identify the minimal rate at which the unit could discharge action potentials repetitively. This procedure was repeated three times, and the average of the three trials was calculated. The subject then increased the abduction force gradually and linearly to a target force that was twice the value associated with the minimal repetitive discharge rate. The recruitment threshold of the motor unit was characterized as the force at which the motor unit began to discharge action potentials repetitively during the ramp increase in the abduction force. This task was repeated three times, and the average of the three trials was defined as the recruitment threshold of the motor unit. 

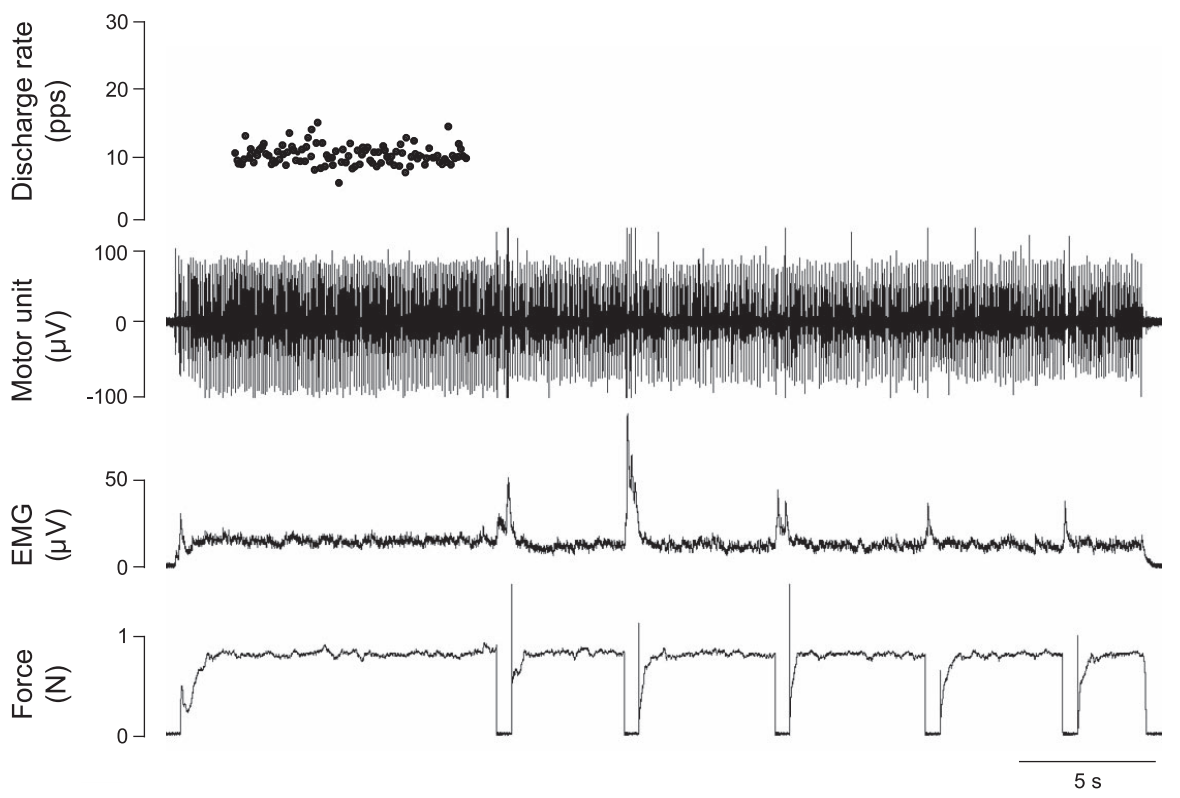

Fig. 2. Representative recordings during the constant-force task, which comprised an initial steady contraction $(10 \mathrm{~s})$ followed by 5 muscle stretches. The traces correspond to the instantaneous discharge rate of an isolated motor unit (trace at top), intramuscular motor unit recordings (second trace from top), rectified interference electromyogram (EMG; third trace from top) of the first dorsal interosseus muscle, and abduction force at the proximal interphalangeal joint of the index finger (trace at bottom). Discharge rate was assessed only during the 10-s steady contraction before the muscle stretches. pps, Pulses/s.
Constant-force task. A target force was set at 2.5\% MVC above the recruitment threshold of the identified motor unit (20). The subject was provided with visual feedback of the force and asked to increase the abduction force to the target force gradually and to sustain it at this value as steadily as possible for $10 \mathrm{~s}$ (Fig. 2). After $10 \mathrm{~s}$, the muscle was stretched rapidly five times with the torque motor at randomized intervals (3-5 s) as the subject continued to produce the constant force. The 15-mm stretches were applied in $20 \mathrm{~ms}$, yielding an average stretch velocity of $\sim 15 \mathrm{rad} / \mathrm{s}\left(\sim 850^{\circ} / \mathrm{s}\right)$ and average displacement of $\sim 0.3 \mathrm{rad}\left(\sim 26^{\circ}\right)$. The subject was instructed not to resist the movement but to regain the target force as soon as possible after each stretch. There was a rest period of $30 \mathrm{~s}$ between each of the 10 constant-force trials.

After completion of the initial 10 trials (Before trials), vibration was applied to the relaxed muscle for $30 \mathrm{~min}$ at an intensity that produced a tonic vibration reflex. A tonic vibration reflex was induced to ensure that direct muscle vibration activated the Ia afferents in the first dorsal interosseus muscle. The distal end of the vibrator (11-mm diameter) was positioned on the skin over the belly of the first dorsal interosseus muscle with the force level of $0.4 \mathrm{~N}$. The vibration frequency was $75 \mathrm{~Hz}$, and the amplitude was adjusted to produce a stable tonic vibration reflex as determined from the changes in EMG amplitude ( $<5 \%$ MVC). Subjects were instructed to ignore their hand during vibration and were distracted by one of the investigators. Immediately after vibration, two sets of 10 steady contractions (as described above) were performed with applied stretches. Each set of 10 contractions took about 10 min to complete and there was a 30-s interval between the first set of 10 contractions (After trials) and the second set (Recovery trials). Subjects in the Control group performed the same protocol, but did not receive the vibration.

\section{Data Analysis}

Force and EMG. The peak force and the average of the full-wave rectified EMG (AEMG) during the MVC were determined for a 0.5-s window centered at the peak force. Force and surface EMG during the 10 -s steady contraction (before the stretches) were used to measure the force fluctuations. The force signal was detrended by subtracting the best-fit linear regression from the signal so that the calculated SD of force reflected variations around the mean and not slow, linear changes over time. The data for the constant-force task were averaged across the 10 trials for the Before, After, and Recovery periods.

Motor units. Single motor unit recordings during the 10-s constantforce task were analyzed for the Vibration group. Action potentials discharged by single motor units were discriminated using a computerized, spike-sorting algorithm (Spike2, version 5.05; Cambridge Electronic Design) that identified the action potentials belonging to a particular motor unit based on waveform amplitude, duration, and shape. In the identification procedure, the amplitude difference was allowed to vary up to $15 \%$, and at least $65 \%$ of the waveform was required to be similar for an action potential to be categorized as belonging to a particular motor unit template. The interspike intervals of identified motor units were examined for every trial. The mean and coefficient of variation of the detrended interspike intervals were determined using custom-designed software written in MatLab (The Mathworks), and they were averaged across trials for the Before, After, and Recovery periods.

Stretch reflex. The three components (short, medium, and long latency) of the stretch reflex were identified on the basis of time to onset (latency). First, surface EMG signals were full-wave rectified and averaged using the peak in index finger acceleration during each stretch as the trigger event (150 stretches). The latency and duration of each reflex component (Fig. 3) were then determined by visual inspection according to previously reported ranges (2). Second, the mean amplitude of each component of the stretch reflex was derived from the full-wave rectified EMG signal that was averaged across 50 stretches each for the Before, After, and Recovery periods. Background EMG was calculated as the mean amplitude for the 100-ms period preceding the onset of muscle stretch, and the mean amplitude of each reflex component was quantified as the mean amplitude of EMG response above the background EMG. The mean amplitude of each reflex component was further normalized to the value obtained before vibration.

Dependent variables. The dependent variables for the MVC task were the peak force and the AEMG. The dependent variables for the constant-force task were the SD of force, AEMG, and the mean and coefficient of variation for interspike intervals of the motor units during the steady contraction, and the mean EMG amplitude for the background and three components of the stretch reflex during the Before, After, and Recovery periods.

\section{Statistical Analysis}

The laterality quotient, the target force, and the latency and duration for the stretch reflex components were compared between groups using an unpaired $t$-test. For each group, the peak force and EMG during the MVC task were compared with a one-factor ANOVA (start 


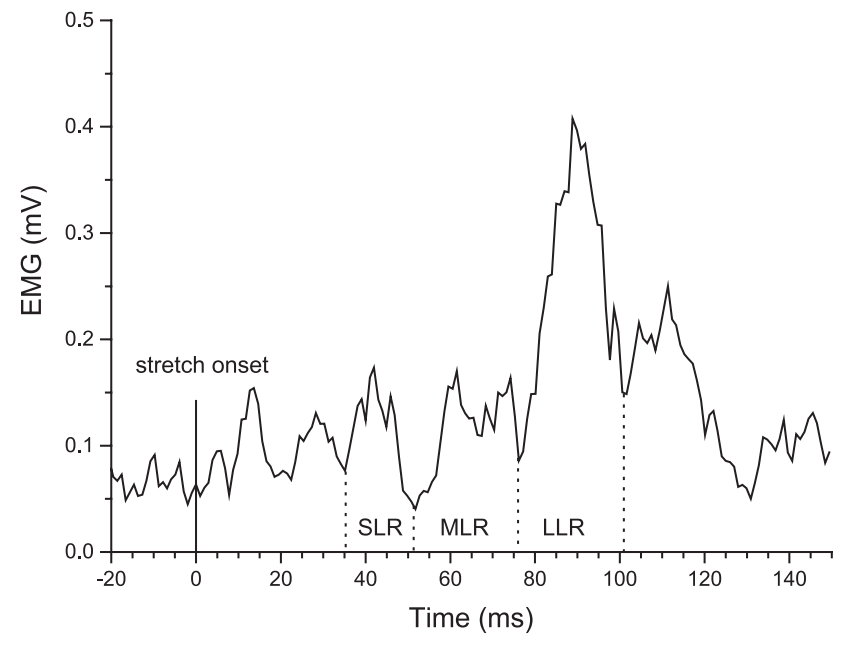

Fig. 3. Representative stretch reflexes evoked in the first dorsal interosseus muscle and averaged across 150 trials. Rectified EMG signals were aligned to the onset of muscle stretch for averaging. The onset of muscle stretch (time 0 ) was detected from the acceleration of the index finger. Vertical dashed lines denote the short-(SLR), medium- (MLR), and long-(LLR) latency components of the stretch reflex.

and end of the experiment) with repeated measures. The background EMG and the mean EMG amplitude for each stretch reflex component, the SD of force, AEMG, mean interspike intervals, and the coefficient of variation for the interspike intervals during the constantforce task were compared with one-factor ANOVAs (Before, After, and Recovery periods) with repeated measures. An alpha level of 0.05 was chosen for all statistical comparisons, and post hoc comparisons (Newman-Keuls) were performed when significance was found. $P<$ 0.05 or $P<0.01$ was indicated when significance was found. Unless stated otherwise, the data are presented as means \pm SD in the text and tables and as means $\pm \mathrm{SE}$ in the figures.

\section{RESULTS}

The study examined the influence of prolonged muscle vibration on the amplitude of the stretch reflex, the discharge rate of motor units, and the fluctuations in force during brief isometric contractions of the first dorsal interosseus muscle. Vibration reduced the MVC force by $15 \%(P<0.01)$ and maximal EMG by $14 \%(P<0.05$; Table 1$)$. In contrast, there was no change in the MVC force $(P=0.27)$ or maximal EMG $(P=0.74)$ over the course of the experiment for subjects in the Control group. The recruitment thresholds of the motor units averaged $2.6 \pm 2.6 \%$ MVC (range: $0.19-9.49 \%$ MVC force), and the target force during the constant-force task was $5.1 \pm$

Table 1. Peak force and maximal EMG during the MVC task at the start and end of the experiment

\begin{tabular}{lcc}
\hline \hline & Start & End \\
\hline Vibration group & & \\
Peak force, $\mathrm{N}$ & $34.5 \pm 10.0$ & $28.7 \pm 9.4 \dagger$ \\
Maximal EMG, mV & $0.381 \pm 0.162$ & $0.327 \pm 0.141^{*}$ \\
Control group & & \\
Peak force, $\mathrm{N}$ & $32.0 \pm 9.1$ & $30.0 \pm 9.5$ \\
Maximal EMG, mV & $0.456 \pm 0.153$ & $0.441 \pm 0.158$ \\
\hline
\end{tabular}

Values are means \pm SD. EMG, electromyogram; MVC, maximal voluntary contraction. $* P<0.05, \dagger P<0.01$ compared with the data at the start of the protocol.
Table 2. Time to onset and duration of the short-, medium-, and long-latency components of the stretch reflex

\begin{tabular}{lll}
\hline \hline & Vibration & Control \\
\hline Time to onset & & \\
Short latency & $29.6 \pm 4.9$ & $29.7 \pm 4.3$ \\
Medium latency & $49.5 \pm 6.5$ & $50.0 \pm 4.0$ \\
$\quad$ Long latency & $79.2 \pm 10.7$ & $81.9 \pm 10.4$ \\
Duration & & \\
$\quad$ Short latency & $19.9 \pm 3.6$ & $20.3 \pm 2.4$ \\
Medium latency & $29.7 \pm 6.7$ & $31.9 \pm 8.9$ \\
Long latency & $25.8 \pm 4.2$ & $28.3 \pm 8.0$ \\
\hline
\end{tabular}

Values are means \pm SD given in ms averaged across all periods (before vibration, after vibration, and during recovery).

$2.6 \%$ MVC for the Vibration group and $4.8 \pm 1.6 \%$ MVC for the Control group $(5.0 \pm 2.3 \% \mathrm{MVC}$ across groups; $P=0.72)$.

\section{Stretch Reflex}

Onset latency and duration of the reflex components did not differ between the groups (Table 2). In the Vibration group, the amplitude of the short-latency reflex increased significantly after vibration during both the After and Recovery periods $(P<0.05$ for both; Table 3$)$. When the values were normalized to those obtained before vibration, the relative increases were $33 \%$ during the After period and 38\% during the Recovery period $(P<0.01$ for both). There was no evidence of recovery during the 20 trials after vibration because there was no difference between the values during the After and Recovery periods $(P=0.65)$. The amplitude of the medium- and longlatency components of the stretch reflex did not change with the intervention $(P>0.41)$. Although subjects matched the same target force before and after vibration, there was a small increase $(\sim 7 \% ; P<0.05)$ in the background EMG after vibration (Table 4). Subjects in the Control group did not experience any change in the amplitude of the stretch reflex $(P>0.23)$ or the background EMG $(P=0.99)$.

Table 3. Amplitude of the stretch reflex components before vibration, after vibration, and during recovery

\begin{tabular}{llll}
\hline \hline & \multicolumn{1}{c}{ Before } & \multicolumn{1}{c}{ After } & Recovery \\
\hline Vibration group & & & \\
$\quad$ Short latency, $\mu \mathrm{V}$ & $35.23 \pm 27.29$ & $44.54 \pm 35.78^{*}$ & $44.28 \pm 36.40 *$ \\
Medium latency, $\mu \mathrm{V}$ & $22.97 \pm 17.93$ & $21.28 \pm 14.40$ & $22.53 \pm 16.27$ \\
Long latency, $\mu \mathrm{V}$ & $58.49 \pm 51.91$ & $52.15 \pm 42.13$ & $46.70 \pm 36.79$ \\
Short latency, $\%$ & $100.0 \pm 0.0$ & $133.3 \pm 46.0 \dagger$ & $138.3 \pm 54.5 \dagger$ \\
Medium latency, $\%$ & $100.0 \pm 0.0$ & $101.0 \pm 34.1$ & $103.9 \pm 42.3$ \\
Long latency, $\%$ & $100.0 \pm 0.0$ & $106.2 \pm 45.3$ & $95.1 \pm 34.6$ \\
Control group & & & \\
Short latency, $\mu \mathrm{V}$ & $40.12 \pm 22.75$ & $42.80 \pm 24.03$ & $42.80 \pm 27.85$ \\
Medium latency, $\mu \mathrm{V}$ & $22.88 \pm 16.05$ & $27.13 \pm 18.38$ & $26.23 \pm 19.20$ \\
Long latency, $\mu \mathrm{V}$ & $56.59 \pm 48.06$ & $56.64 \pm 41.62$ & $49.63 \pm 35.34$ \\
Short latency, $\%$ & $100.0 \pm 0.0$ & $108.0 \pm 23.5$ & $103.4 \pm 13.0$ \\
Medium latency, $\%$ & $100.0 \pm 0.0$ & $117.4 \pm 33.0$ & $115.0 \pm 47.2$ \\
Long latency, $\%$ & $100.0 \pm 0.0$ & $106.7 \pm 42.2$ & $93.8 \pm 31.0$ \\
\hline
\end{tabular}

Values are means \pm SD. Stretch reflex amplitude was averaged across 10 trials before vibration (Before), 10 trials after vibration (After), and 10 trials during recovery (Recovery). Data are reported as absolute values $(\mu \mathrm{V})$ and relative $(\%)$ to those Before vibration. ${ }^{*} P<0.05, \dagger P<0.01$ compared with before vibration. 
Table 4. Background EMG before muscle stretches during the constant-force task before vibration, after vibration, and during recovery

\begin{tabular}{lcll}
\hline \hline & Before & \multicolumn{1}{c}{ After } & \multicolumn{1}{c}{ Recovery } \\
\hline Vibration group & $41.2 \pm 23.3$ & $43.7 \pm 23.2^{*}$ & $43.9 \pm 23.1^{*}$ \\
Control group & $43.7 \pm 14.5$ & $43.9 \pm 15.1$ & $43.6 \pm 15.8$ \\
\hline
\end{tabular}

Values are means $\pm \mathrm{SD}$ given in $\mu \mathrm{V}$. $* P<0.05$ compared with before vibration.

\section{Force Fluctuations}

The SD of force during the steady contractions increased significantly after vibration by $21 \%$ during the After period $(P<0.01)$ and by $28 \%$ during the Recovery period $(P<0.01)$ relative to the values obtained before vibration $(57.1 \pm 18.3$ $\mathrm{mN}$; Fig. 4). The SD of force did not differ $(P=0.29)$ during the After $(69.1 \pm 25.2 \mathrm{mN})$ and Recovery $(72.9 \pm 30.0 \mathrm{mN})$ periods. The AEMG during the steady contractions also increased by $9-10 \%$ after vibration $(P<0.05$; Fig. 4). Subjects in the Control group did not experience any change in the SD of force $(58.3 \pm 21.8 \mathrm{mN} ; P=0.29)$ or AEMG $(P=0.85)$ across the protocol.

\section{Motor Unit Discharge}

Because of the technical challenges of recording the same motor unit both before and after prolonged muscle vibration, the data set was limited to 16 motor units that were successfully followed across all three periods. The mean discharge rate of the motor units increased significantly from $10.3 \pm 2.7$ pulses/s (pps) before vibration to $12.2 \pm 3.1 \mathrm{pps}(P<0.01)$ during the After period and $11.9 \pm 2.6 \mathrm{pps}(P<0.01)$ during the Recovery period (Fig. 5). There was no difference in the mean discharge rate between the two periods after vibration $(P=0.47)$. Discharge rate variability, quantified as the coefficient of variation for the interspike interval, was $\sim 26 \%$ and
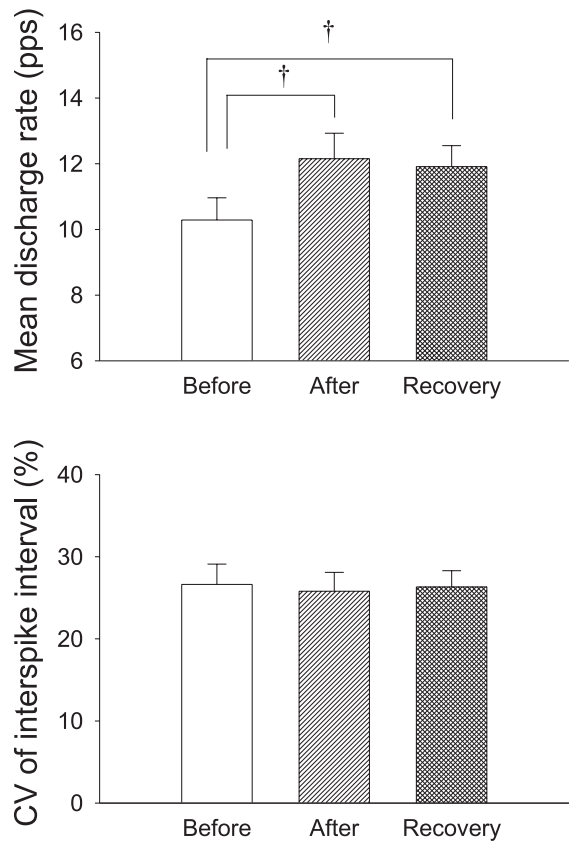

Fig. 5. Mean and coefficient of variation (CV) for interspike intervals of motor units $(n=16)$ before and after vibration. Mean discharge rate (pps) was determined from the reciprocal of the mean interspike interval. Values are means \pm SE. ${ }^{\dagger} P<0.01$.

did not change across periods $(26.6 \pm 9.9 \%$ before vibration, $25.8 \pm 9.2 \%$ after vibration, and $26.3 \pm 7.9 \%$ during recovery; $P=0.94)$.

\section{DISCUSSION}

The purpose of this study was to compare the influence of prolonged vibration of a hand muscle on the amplitude of the stretch reflex, motor unit discharge rate, and force fluctuations during steady, submaximal contractions. In contrast to our
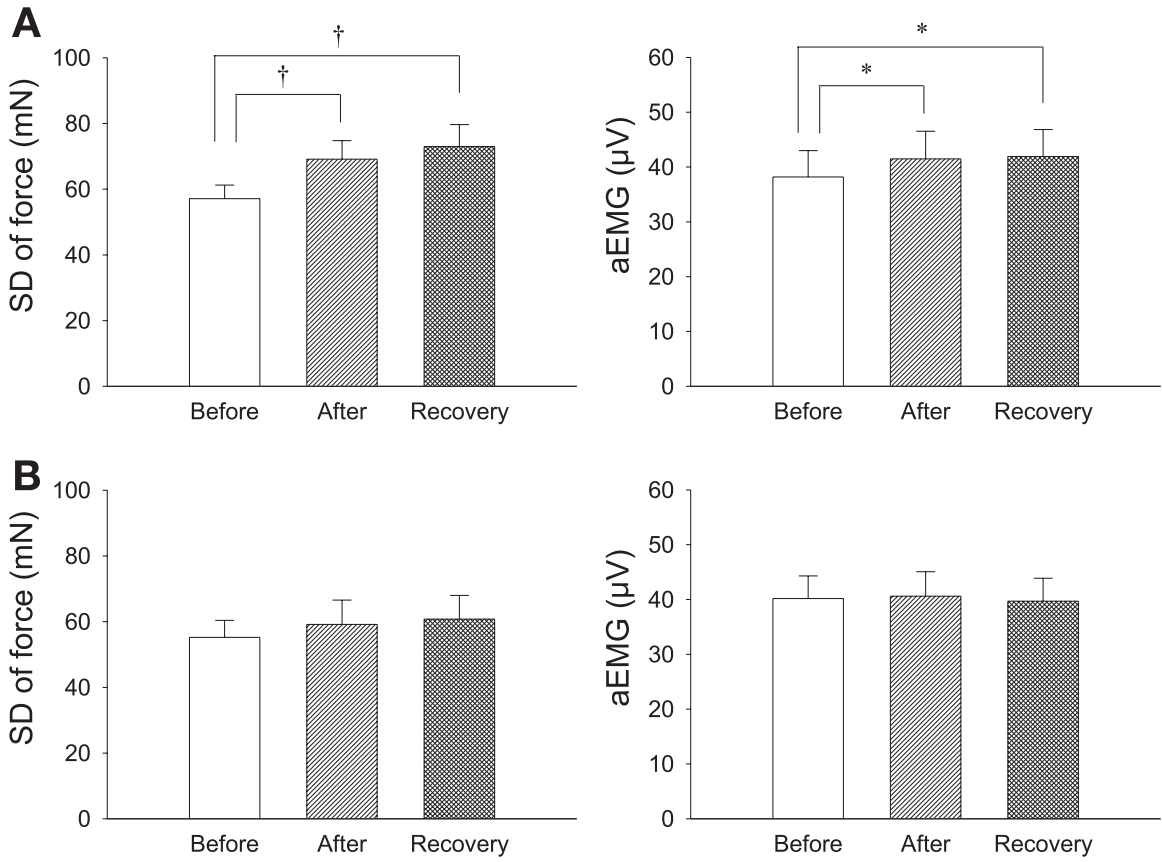

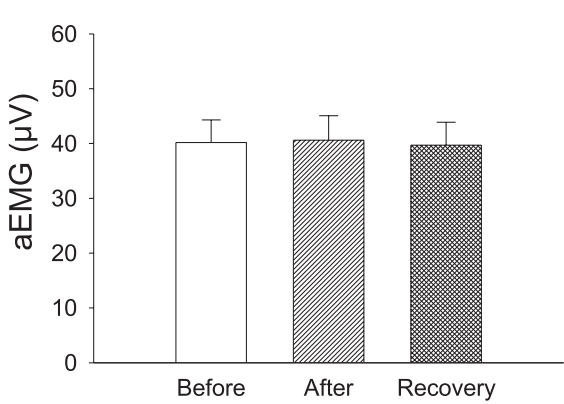

Fig. 4. Average of the full-wave rectified (AEMG) and the SD of force during the constant-force task before and after vibration. The data are averaged across 10 trials before vibration (Before), 10 trials after vibration (After), and 10 trials during recovery (Recovery) for the Vibration group $(n=20)$ and the Control group $(n=12)$. Values are means \pm SE. ${ }^{*} P<0.05 .{ }^{\dagger} P<0.01$. 
expectations, prolonged vibration increased the force fluctuations, the amplitude of the short-latency stretch reflex, and motor unit discharge rate, but it did not increase discharge rate variability. Subjects in the Control group did not experience any changes in the force fluctuations or stretch reflexes across the protocol.

\section{Stretch Reflex}

The increase in the amplitude of the short-latency component of the stretch reflex in the first dorsal interosseus muscle contrasts with the frequently observed depression of H-reflex amplitude after vibration $(1,6,11,38)$. This result, however, is consistent with other reports in the literature. For example, van Boxtel (39) observed a marked increase in the amplitude of the stretch reflex and depression of the H-reflex amplitude in the soleus muscle after 10- to 120 -s vibration, with a slower recovery as vibration was prolonged. Furthermore, Heckman et al. (11) reported that $20 \mathrm{~min}$ of vibration caused a $22-58 \%$ increase in stretch-reflex amplitude and a 55-100\% decrease in H-reflex amplitude in the soleus muscle, and these changes persisted for 15-30 min. In the present study, the amplitude of the short-latency component of the stretch reflex increased by $33-38 \%$ after $30 \mathrm{~min}$ of vibration, and this increase lasted for at least $20 \mathrm{~min}$.

Differential changes in the amplitudes of the $\mathrm{H}$ and stretch reflexes are typically interpreted to indicate a change in the sensitivity of the muscle spindle before and after an intervention $(8,27)$. In contrast to the electrical stimulation that is used to elicit the $\mathrm{H}$ reflex, stretch of the muscle to evoke a stretch reflex involves the muscle spindle, and the magnitude of the response depends on the sensitivity of the muscle spindles, which is determined by the level of fusimotor activity. In the present study, the small $(\sim 7 \%)$ increase in the background EMG after vibration may indicate enhanced fusimotor drive to the muscle spindles ( $\alpha-\gamma$ coactivation) to achieve the target force due to the vibration-induced decline in the force capacity of the muscle. Alternatively, fusimotor drive to the muscle spindles may have been selectively enhanced $(15,27,29)$ after vibration.

The apparent discrepancy between the $\mathrm{H}$ and stretch reflexes, however, may not be simply due to a difference in the sensitivity of the muscle spindle. Other factors may also be involved, such as different sensitivities of electrically and mechanically evoked reflexes to presynaptic inhibition. In support of this view, Morita et al. (25) reported that the depression of the reflex response by a conditioning stimulation in the soleus muscle was less in the Achilles tendon tap compared with the $\mathrm{H}$ reflex. These authors suggested that this apparent difference in the effect of presynaptic inhibition was probably due to a difference in the composition or temporal dispersion of the afferent volleys evoked by electrical and mechanical stimuli.

Furthermore, the present study tested the stretch reflex in a hand muscle, whereas most previous reports examined the soleus muscle. Compared with the triceps surae muscle, the short-latency reflex is suppressed in the first dorsal interosseus muscle (37), probably by inhibitory input from higher centers. The absence of an increase in the medium- and long-latency reflexes in the present study may also be a consequence of inhibition in higher centers. Hence, it is possible that the vibration-induced modulation of the spinal reflexes can vary with the muscles under study. Without H-reflex data in the hand muscle after prolonged vibration, however, this possibility cannot be addressed with the present data. Nonetheless, the increase in the amplitude of the short-latency component of the stretch reflex after vibration indicates that excitatory synaptic input to $\alpha$-motor neurons increased during the constant-force task after $30 \mathrm{~min}$ of vibration.

\section{Motor Unit Activity and Force Fluctuations}

Because the amplitude of the surface-detected EMG underestimates the motor output from the spinal cord $(10,16)$, the significant increase in AEMG after vibration indicates that greater motor unit activity was necessary to achieve the target force during the constant-force task. Accordingly, mean discharge rate of the discriminated motor units increased significantly during the trials performed after vibration. The enhanced motor unit activity after vibration is likely attributable to the reduced force capacity of the muscle after prolonged vibration. In the present study, a tonic vibration reflex $(<5 \%$ MVC) was induced for $30 \mathrm{~min}$ to ensure that vibration activated the group Ia afferents, which resulted in $\sim 15 \%$ reduction in MVC force. Because the short-latency component of the stretch reflex was increased after prolonged vibration, the reduction in MVC force cannot be due to the withdrawal of Ia afferent support for the voluntary contraction. It is likely that the prolonged tonic vibration reflex induced a failure in the excitation-contraction coupling that is known to occur during prolonged low-force contractions (9). The magnitude of the decline in the MVC force was similar to the reduction in the MVC force $(\sim 15 \%)$ that was observed after a prolonged voluntary contraction $(60 \mathrm{~min})$ as low as $2.5 \% \operatorname{MVC}(18,19)$. Although MVC force decreased by $19 \%$ after 30 min of the Achilles tendon vibration (40), the reduction could not have been due to a failure in the excitation-contraction coupling because a tonic vibration reflex was not induced in that study. Nonetheless, the increase in discharge rate $(16-18 \%)$ observed in the present study is consistent with the increase $(21 \%)$ that was observed in the supraspinatus muscle during recovery from the 30-min contraction at 11-12\% MVC force (14).

Alternatively, the greater AEMG activity during the constant-force trials after vibration may have been necessary to overcome heightened activity in the antagonist muscle (second palmar interosseus). For example, 30 min of vibration of the distal wrist flexor tendons have been shown to enhance corticospinal excitability in the antagonist muscle (extensor carpi radialis brevis) that persisted for $60 \mathrm{~min}$ (36). Because the EMG activity of second palmar interosseus was not recorded in the present study, the increase in AEMG during the constantforce trials after vibration may have been necessary to accommodate both the reduced force capacity of the agonist muscle and the increased activation of the antagonist muscle.

The increases in AEMG and discharge rate during the constant-force trials after vibration were accompanied by an increase in the force fluctuations (SD of force). In contrast, there was no change in discharge rate variability, which is known to have a profound influence on force fluctuations (17, $20,26)$. Because the SD of force increases monotonically with contraction intensity in the low-to-moderate force range ( $\leq 60 \%$ MVC) $(5,23,24,26)$, the greater force fluctuations 
after vibration were likely caused by the increase in motor unit activity, perhaps including the recruitment of higher threshold motor units that discharged at low rates. This possibility is consistent with observations that used other interventions. For example, Shinohara et al. (34) found that 20 days of bed rest reduced the MVC force of leg muscles and that increases in EMG activity when performing steady contractions of plantar flexor or knee extensor muscles were accompanied by greater force fluctuations. Similarly, Hunter and Enoka (13) found that practice-induced adaptations in the time to failure for a fatiguing contraction produced parallel changes in EMG activity and force fluctuations. In contrast, enhanced coactivation of the antagonist muscle does not appear to influence the force fluctuations (4). Hence, the increase in the force fluctuations during the steady contractions performed after vibration appears to be attributable to the increase in motor unit activity.

The present finding that vibration increased the force fluctuations during steady contractions with a hand muscle contrasts with previous reports of a reduction in the force fluctuations for the plantar flexor muscles $(6,40)$. For example, Yoshitake et al. (40) found that a decrease in MVC force caused by 30 min of vibration applied to the Achilles tendon was accompanied by a reduction in the force fluctuations during steady contractions at $2.5-10 \% \mathrm{MVC}$ force. One possibility is that the muscle spindle may respond differently to muscle vibration in the first dorsal interosseus muscle compared with tendon vibration in the plantar flexor muscles: all muscle spindles may not be influenced uniformly within the first dorsal interosseus muscle during muscle vibration. Furthermore, the location of the vibrator (muscle vs. tendon) may have involved different populations of sensory receptors. Alternatively, although the steady contractions with the plantar flexor muscles after prolonged vibration did not require an increase in EMG activity for the three muscles (medial and lateral gastrocnemius and soleus), the changes in motor unit activity (mean and coefficient of variation) may have been too subtle to be detected with surface electrodes. Motor unit recordings from the plantar flexor muscles are necessary to resolve the apparent discrepancy between the observations on hand and leg muscles.

In conclusion, prolonged vibration of a hand muscle increased the amplitude of the short-latency component of the stretch reflex, the discharge rate of motor units, and the force fluctuations during steady, submaximal contractions. These adjustments were likely necessary to perform the constantforce task due to the vibration-induced decrease in the force capacity of the muscle. These results suggest that the influence of Ia afferent input on force fluctuations during steady contractions appears to depend on the location of the vibration and the muscles involved in the intervention.

\section{ACKNOWLEDGMENTS}

The authors acknowledge the assistance of Alain Frigon with the experiments and Carol Mottram for comments on a draft of the manuscript.

\section{GRANTS}

This work was supported by an award from the Nakayama Foundation for Human Science (to M. Shinohara), National Institute of Neurological Disorders and Stroke Grant NS-42734 and National Institute on Aging Grant AG-09000 (to R. M. Enoka).

\section{REFERENCES}

1. Abbruzzese M, Minatel C, Reni L, and Favale E. Postvibration depression of the H-reflex as a result of a dual mechanism: an experimental study in humans. J Clin Neurophysiol 18: 460-470, 2001.

2. Balestra $\mathbf{C}$, Duchateau J, and Hainaut $\mathbf{K}$. Effects of fatigue on the stretch reflex in a human muscle. Electroencephalogr Clin Neurophysiol 85: 46-52, 1992.

3. Burke D, Hagbarth KE, Lofstedt L, and Wallin BG. The responses of human muscle spindle endings to vibration of non-contracting muscles. J Physiol 261: 673-693, 1976.

4. Burnett RA, Laidlaw DH, and Enoka RM. Coactivation of the antagonist muscle does not covary with steadiness in old adults. $J$ Appl Physiol 89: 61-71, 2000.

5. Christou EA and Carlton LG. Old adults exhibit greater motor output variability than young adults only during rapid discrete isometric contractions. J Gerontol A Biol Sci Med Sci 56: B524-B532, 2001.

6. Cresswell AG and Löscher WN. Significance of peripheral afferent input to the alpha-motoneurone pool for enhancement of tremor during an isometric fatiguing contraction. Eur J Appl Physiol 82: 129-136, 2000.

7. Eklund $\mathbf{G}$ and Hagbarth KE. Normal variability of tonic vibration reflexes in man. Exp Neurol 16: 80-92, 1966.

8. Enoka RM, Hutton RS, and Eldred E. Changes in excitability of tendon tap and Hoffmann reflexes following voluntary contractions. Electroencephalogr Clin Neurophysiol 48: 664-672, 1980.

9. Enoka RM and Stuart DG. Neurobiology of muscle fatigue. $J$ Appl Physiol 72: 1631-1648, 1992.

10. Farina D, Merletti R, and Enoka RM. The extraction of neural strategies from the surface EMG. J Appl Physiol 96: 1486-1495, 2004.

11. Heckman CJ, Condon SM, Hutton RS, and Enoka RM. Can Ib axons be selectively activated by electrical stimuli in human subjects? Exp Neurol 86: 576-582, 1984.

12. Hultborn H, Illert M, Nielsen J, Paul A, Ballegaard M, and Wiese H. On the mechanism of the post-activation depression of the H-reflex in human subjects. Exp Brain Res 108: 450-462, 1996.

13. Hunter SK and Enoka RM. Changes in muscle activation can prolong the endurance time of a submaximal isometric contraction in humans. J Appl Physiol 94: 108-118, 2003.

14. Jensen BR, Pilegaard M, and Sjøgaard G. Motor unit recruitment and rate coding in response to fatiguing shoulder abductions and subsequent recovery. Eur J Appl Physiol 83: 190-199, 2000.

15. Jones KE, Wessberg J, and Vallbo Å. Proprioceptive feedback is reduced during adaptation to a visuomotor transformation: preliminary findings. Neuroreport 12: 4029-4033, 2001.

16. Keenan KG, Farina D, Maluf KS, Merletti R, and Enoka RM. Influence of amplitude cancellation on the simulated surface electromyogram. J Appl Physiol 98: 120-131, 2005.

17. Kornatz KW, Christou EA, and Enoka RM. Practice reduces motor unit discharge variability in a hand muscle and improves manual dexterity in old adults. J Appl Physiol 98: 2072-2080, 2005.

18. Kouzaki M, Shinohara M, Masani K, and Fukunaga T. Force fluctuations are modulated by alternate muscle activity of knee extensor synergists during low-level sustained contraction. J Appl Physiol 97: 21212131, 2004.

19. Kouzaki M, Shinohara M, Masani K, Tachi M, Kanehisa H, and Fukunaga T. Local blood circulation among knee extensor synergists in relation to alternate muscle activity during low-level sustained contraction. J Appl Physiol 95: 49-56, 2003.

20. Laidlaw DH, Bilodeau M, and Enoka RM. Steadiness is reduced and motor unit discharge is more variable in old adults. Muscle Nerve 23: 600-612, 2000.

21. Lance JW, Burke D, and Andrews CJ. The reflex effects of muscle vibration. In: New Developments in Electromyography and Clinical Neurophysiology, edited by Desmedt JE. Basel: Karger, 1973, p. 444-462.

22. Li ZM, Pfaeffle HJ, Sotereanos DG, Goitz RJ, and Woo SL. Multidirectional strength and force envelope of the index finger. Clin Biomech (Bristol, Avon) 18: 908-915, 2003.

23. Löscher WN, Cresswell AG, and Thorstensson A. Electromyographic responses of the human triceps surae and force tremor during sustained submaximal isometric plantar flexion. Acta Physiol Scand 152: 73-82, 1994

24. Löscher WN and Gallasch E. Myo-electric signals from two extrinsic hand muscles and force tremor during isometric handgrip. Eur J Appl Physiol 67: 99-105, 1993. 
25. Morita H, Petersen N, Christensen LO, Sinkjaer T, and Nielsen J. Sensitivity of $\mathrm{H}$-reflexes and stretch reflexes to presynaptic inhibition in humans. J Neurophysiol 80: 610-620, 1998.

26. Moritz CT, Barry BK, Pascoe MA, and Enoka RM. Discharge rate variability influences the variation in force fluctuations across the working range of a hand muscle. J Neurophysiol 93: 2449-2459, 2005.

27. Nafati G, Rossi-Durand C, and Schmied A. Proprioceptive control of human wrist extensor motor units during an attention-demanding task. Brain Res 1018: 208-220, 2004.

28. Oldfeld RC. The assessment and analysis of handedness: the Edinburgh inventory. Neuropsychologia 9: 97-113, 1971.

29. Ribot-Ciscar E, Rossi-Durand C, and Roll JP. Increased muscle spindle sensitivity to movement during reinforcement manoeuvres in relaxed human subjects. $J$ Physiol 523: 271-282, 2000.

30. Roll JP, Vedel JP, and Ribot E. Alteration of proprioceptive messages induced by tendon vibration in man: a microneurographic study. Exp Brain Res 76: 213-222, 1989.

31. Shinohara M, Keenan KG, and Enoka RM. Contralateral activity in a homologous hand muscle during voluntary contractions is greater in old adults. J Appl Physiol 94: 966-974, 2003.

32. Shinohara M, Keenan KG, and Enoka RM. Fluctuations in motor output during steady contractions are weakly related across contraction types and between hands. Muscle Nerve, 31: 731-750, 2005.

33. Shinohara M, Moritz CT, Frigon A, and Enoka RM. Vibration-induced enhancement of the stretch reflex is accompanied by an increase in the force fluctuations for a hand muscle (Abstract). Soc Neurosci Abstr 188.8, 2004.

34. Shinohara M, Yoshitake Y, Kouzaki M, Fukuoka H, and Fukunaga T. Strength training counteracts motor performance losses during bed rest. J Appl Physiol 95: 1485-1492, 2003.

35. Spiegel KM, Stratton J, Burke JR, Glendinning DS, and Enoka RM. The influence of age on the assessment of motor unit activation in a human hand muscle. Exp Physiol 81: 805-819, 1996.

36. Steyvers M, Levin O, Van Baelen M, and Swinnen SP. Corticospinal excitability changes following prolonged muscle tendon vibration. $\mathrm{Neu}$ roreport 14: 1901-1905, 2003.

37. Thilmann AF, Schwarz M, Topper R, Fellows SJ, and Noth J. Different mechanisms underlie the long-latency stretch reflex response of active human muscle at different joints. $J$ Physiol 444: 631-643, 1991.

38. Ushiyama J, Masani K, Kouzaki M, Kanehisa H, and Fukunaga T. Difference in aftereffects following prolonged Achilles tendon vibration on muscle activity during maximal voluntary contraction among plantar flexor synergies. J Appl Physiol 98: 1427-1433, 2005.

39. Van Boxtel A. Differential effects of low-frequency depression, vibrationinduced inhibition, and posttetanic potentiation on H-reflexes and tendon jerks in the human soleus muscle. J Neurophysiol 55: 551-568, 1986.

40. Yoshitake Y, Shinohara M, Kouzaki M, and Fukunaga T. Fluctuations in plantar flexion force are reduced after prolonged tendon vibration. J Appl Physiol 97: 2090-2097, 2004.

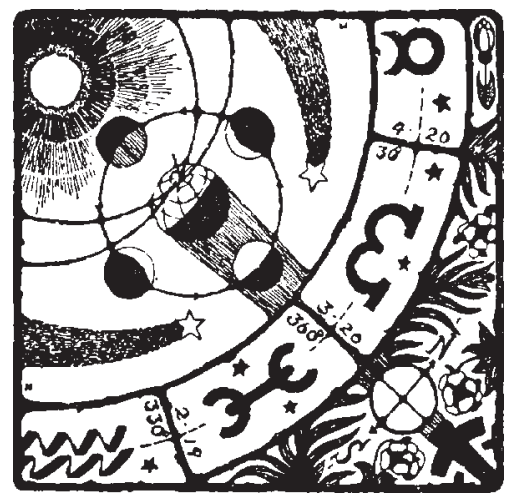

